\title{
Network as a resource: G-lambda project and its architecture
}

\author{
Tomohiro Kudoh ${ }^{1}$ \\ Shuichi Okamoto ${ }^{4,2}$ \\ Yukio Tsukishima ${ }^{3}$, \\ Hideaki Tanaka ${ }^{2}$ \\ Masahiko Jinno ${ }^{3}$
}

\author{
Michiaki Hayashi $^{2}$ \\ Atsuko Takefusa ${ }^{1}$ \\ Tomohiro Otani ${ }^{4,2}$ \\ Atsushi Taniguchi $i^{3,4}$
}

\author{
Akira Hirano ${ }^{3}$ \\ Takahiro Miyamoto ${ }^{2}$ \\ Hidemoto Nakada ${ }^{1}$ \\ Yasunori Sameshima ${ }^{4,3}$
}

1. National Institute of Advanced Industrial Science and Technology (AIST); Tokyo, Japan
\{t.kudoh,atsuko.takefusa, hide-nakada\}@aist.go.jp
2. KDDI R\&D Laboratories; Saitama, Japan
\{mc-hayashi,okamoto,tk-miyamoto,otani,hide\}@kddilabs.jp
3. NTT Network Innovation Laboratories; Kanagawa, Japan
\{hirano.akira,tsukishima.yukio,taniguchi.atsushi,
sameshima.yasunori,jinno.masahiko\}@lab.ntt.co.jp

\begin{abstract}
In computing systems, hardware components such as CPUs, memories and storages are "resources". Resources are managed by resource managers (c.f. a queuing system of a computing center), and a user can use them while they are allocated to the user. To provide a stable service using "resources" connected to a wide area network, network itself should be also considered as a "resource" and bandwidth should be allocated to users appropriately. Resource is provided to a user or upper layer middleware by a resource manager. Therefore, to realize "network as a resource", bandwidth of network should be managed by a network resource manager, and an interface to provide resource service to users or middleware is required. National Institute of Advanced Industrial Science and Technologies (AIST), KDDI R\&D Laboratories, NTT and National Institute of Information and Communications Technology (NICT) are promoting collaborative project, called G-lambda, to establish such a standard interface, which is called GNS-WSI (Grid Network Service-Web Services Interface). In this paper, we will briefly introduce the architecture we assume in the G-lambda project to define GNS-WSI.
\end{abstract}

\section{Keywords}

Network as a resource, bandwidth provisioning, G-lambda, GNSWSI

Permission to make digital or hard copies of all or part of this work for personal or classroom use is granted without fee provided that copies are not made or distributed for profit or commercial advantage and that copies bear this notice and the full citation on the first page. To copy otherwise, or republish, to post on servers or to redistribute to lists, requires prior specific permission and/or a fee.

GridNets 2007 October 17-19, 2007, Lyon, France.

Copyright 2007 ICST ISBN 978-963-9799-07-3.

DOI 10.4108/gridnets.2007.2289

\section{BASIC FUNCTIONS}

Figure 1 shows the basic architecture of G-lambda[1][2]. According to a request from a user or middleware, a resource coordinator communicates with network resource managers (NRM) and compute resource managers (CRM) to make a reservation of a set of resources. Multiple resource coordinators and multiple resource managers can exist in this model. A resource belongs to a domain. A domain has one or some resource managers which manages resources in the domain. In this figure, two network domains exist, and each domain has its own NRM. GNS-WSI is the interface between a resource coordinator and an NRM.

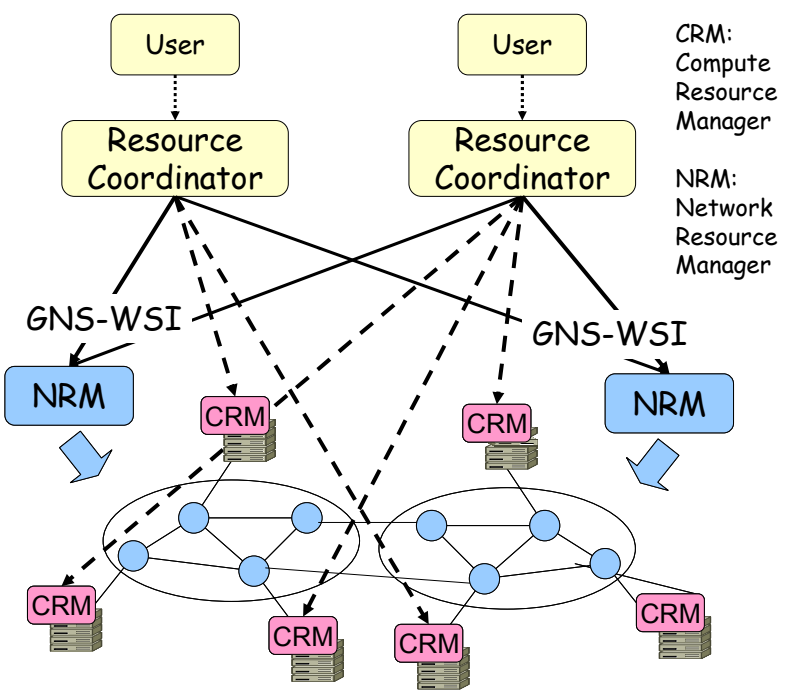

Figure 1. G-lambda basic architecture.

Reserve, release and modify are the basic functions of GNS-WSI. In the reserve operation, bandwidth between two endpoints can be 
reserved. Parameters of the reserve operation include a pair of endpoints, start time and end time of the reservation request, and required bandwidth. An existing reservation can be canceled by the release operation. A reservation can be modified (e.g. shortening/extending reservation period, adding or removing resources) by the modify operation.

\section{2-PHASE COMMIT}

We use 2-phase commit[3] protocol for reserve, release and modify operations. In the 2-phase commit, an operation is made in two phases. At the first phase, a request from a resource coordinator is granted and held if the resource manager can accommodate the request. If anybody says "no" to a request, something of the operation's attempt may need to be changed before committing in the 2 nd phase. If the resource coordinator has sufficient held resources, the resource coordinator commits the held reservation. Figure 2 is the state transition diagram of a state of a resource for each operation.

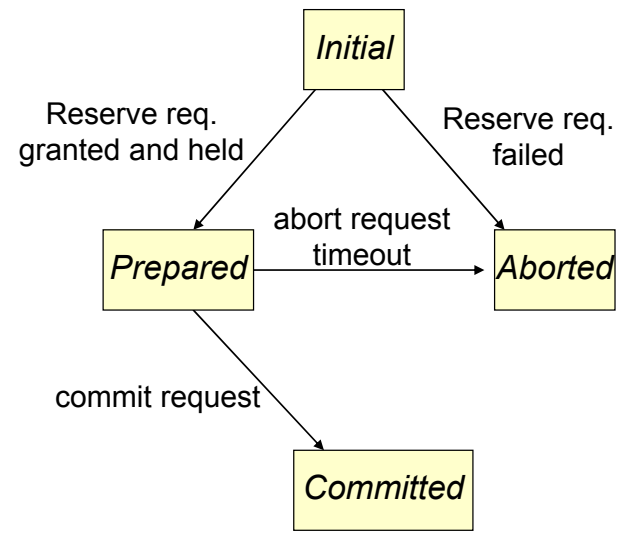

Figure 2. State transition of 2-phase commit.

2-phase commit introduces flexibility for booking multiple resources, and modification of existing reservation can be handled properly.

\section{PER-REQUEST HIERARCHICAL REQUEST MODEL}

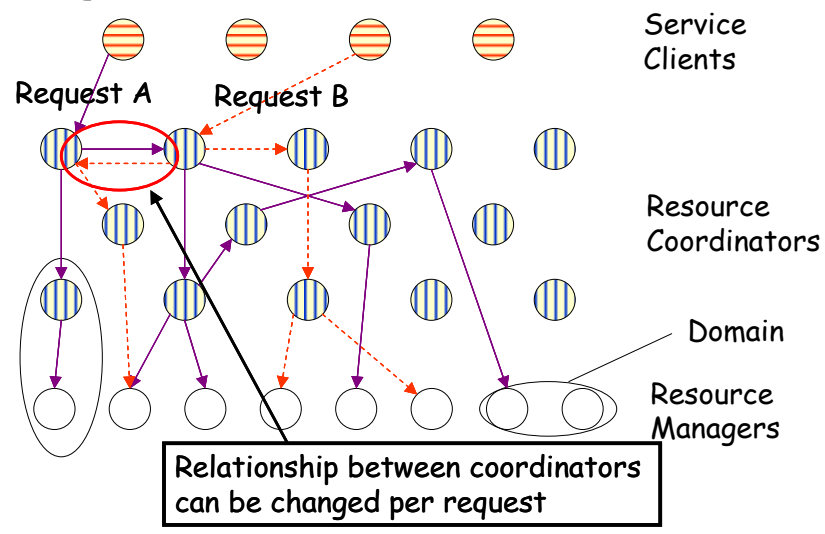

Figure 3. Per-request hierarchical request model
A reservation request from a service client propagates hierarchically through resource coordinators to resource managers, as shown in Figure 3. A request tree is formed for a request. There is no fixed relationship between resource coordinators. A coordinator (namely A) which requested to another coordinator (namely B) may receive a request from B for another request.

This request propagation model is very flexible, and the Chain model [4] can be considered as a subset of this model.

\section{INFORMATION SERVICE}

To find available resources, information such as supported end points and supported bandwidth should be provided. In the Glambda architecture, such information can be provided through information services and/or direct queries to resource managers. An information service can be provided by a network operator, or another service provider (Figure 4).

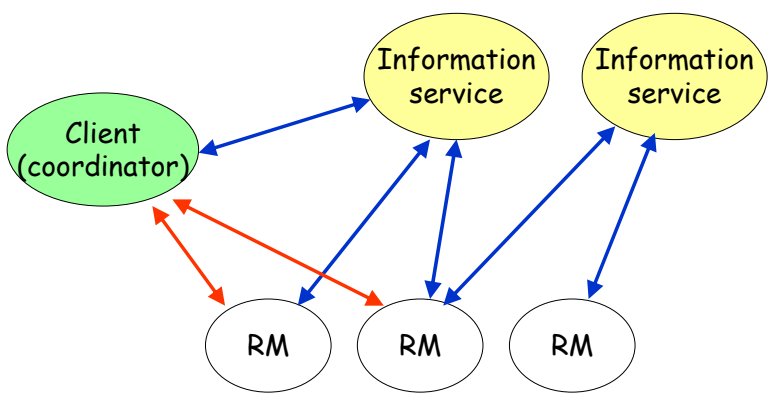

Figure 4. Information service.

For commercial services, network operators may not want to disclose all the topology and availability information of their network. Therefore, each network operator can select information to be provided to a client based on its policy. Policy is determined according to the contract with the client. For example, inquire of availability of all the resources may not be supported. In addition, each network operator can select information services through which the operator provides information to clients. How to make a contract between a user or middleware and a resource manager is out of scope of the G-lambda architecture.

\section{AUTHENTICATION}
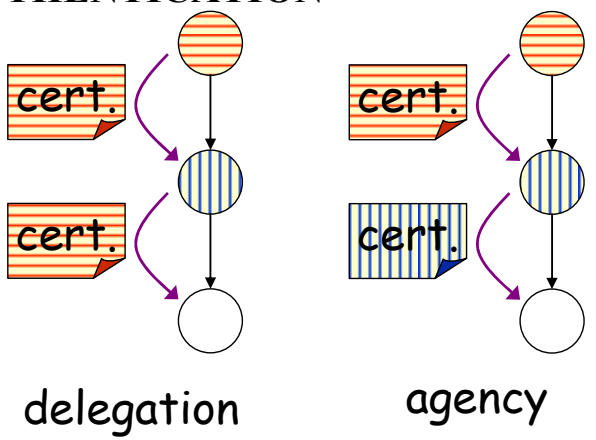

Figure 5.Delegation and Agency.

Certificate based authentication can be used in G-lambda. When a resource coordinator propagates a request form a client to a resource manager or another resource coordinator, we support both delegation and agency models (Figure 5.). In the delegation 
model, a certificate from a client is delegated. In this case, resources are used in the name of the client. In the agency model, a certificate of the coordinator is used. In this case, resources are used in the name of the coordinator. Depending on the functions of the coordinator, an appropriate model can be used.

\section{WEB SERVICES}

Web Services are application components which can be accessed thorough open standard web protocols (XML, SOAP, etc.). Web Services interface enables interaction between application components, and provide very high level interoperability among the components. GNS-WSI is supposed to be used in a enterprise environment. Therefore, GNS-WSI uses WSRF-based Web Services protocol to maximize the interoperability.

Figure 6 shows an example of a set of messages exchanged in a reserve operation. Status change of the NRM is polled by a message different from the request, and thus all the operations are non-blocking.

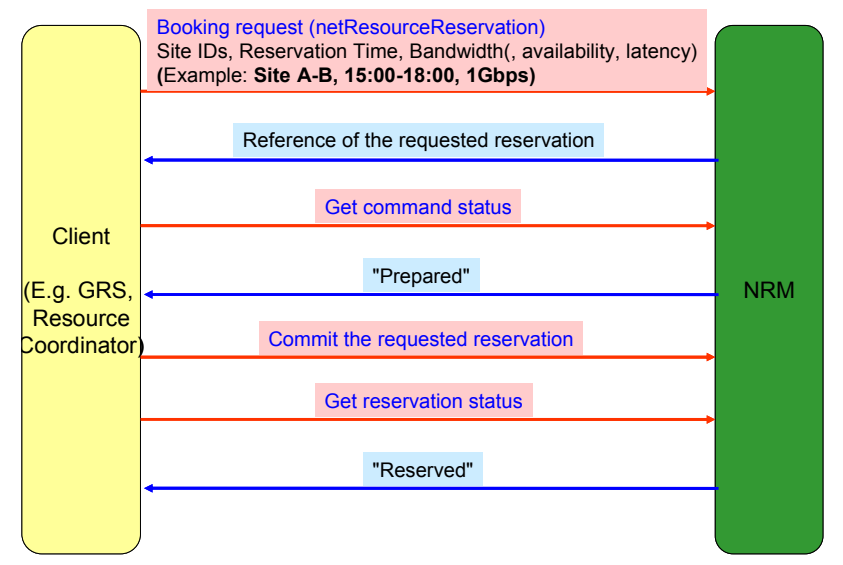

Figure 6. An example of messages of GNS-WSI.

\section{SUMMARY}

In this paper, we briefly introduced the architecture of G-lambda. We are currently discussing on additional parameters of the operations to support SLA and AAA. Operations for information services are also under discussion.

At GLIF2006 and SC06 conferences, we conducted an experiment in cooperation with US EnLIGHTened computing project[5]. In the experiment, .GNS-WSI was used to reserve bandwidth in Japan and US, and we could confirm effectiveness of the interface.

\section{ACKNOWLEDGMENTS}

We would like to thank the G-lambda project members for their variable comments and contributions. We also would like to express our sincere gratitude to the EnLIGHTened computing project team for giving us an opportunity to examine and demonstrate our architecture through joint experiments. The work of AIST is partially funded by the Science and Technology Promotion Program's "Optical Paths Network Provisioning based on Grid Technologies" of MEXT, Japan, which AIST and NTT are jointly promoting.

\section{REFERENCES}

[1] G-lambda Project Website. http://www.g-lambda.net/.

[2] A. Takefusa et al. G-lambda: Coordination of a Grid Scheduler and Lambda Path Service over GMPLS. Future Generation Computing Systems, 22(2006):868-875, 2006.

[3] A. S. Tannenbaum. Distributed Operating Systems, Prentice Hall (1994).

[4] D. Katramatos et al. TeraPaths: End-to-End Network Path QoS Configuration Using Cross-Domain Reservation Negotiation. Proc. of GridNets2006

[5] S. Thorpe et al. G-lambda and EnLIGHTened: Wrapped In Middleware, Co-allocating Compute and Network Resources Across Japan and the US. Proc. of GridNets 2007 (to appear) 